\title{
On $S_{\alpha}^{\beta}(\theta)$-convergence and strong $N_{\alpha}^{\beta}(\theta, p)$-summability
}

\author{
Hacer Şengül \\ Department of Mathematics, Siirt University 56100, Siirt, Turkey.
}

Communicated by P. Kumam

\begin{abstract}
In the papers [M. Et, H. Şengül, Filomat, 28 (2014), 1593-1602] and [H. Şengül, M. Et, Acta Math. Sci. Ser. B Engl. Ed., 34 (2014), 473-482], we defined the spaces of $S^{\alpha}(\theta)$-convergent and strongly $N^{\alpha}(\theta, p)$-summable sequences. In this paper these spaces are generalized to the space of $S_{\alpha}^{\beta}(\theta)$-convergent sequences and the space of strongly $N_{\alpha}^{\beta}(\theta, p)$-summable sequences and are given some inclusion relationships among these spaces. (C)2017 All rights reserved.
\end{abstract}

Keywords: Lacunary sequence, statistical convergence, Cesàro summability. 2010 MSC: 40A05, 40C05, 46A45.

\section{Introduction}

In 1951, Steinhaus [27] and Fast [15] introduced the concept of statistical convergence and later in 1959, Schoenberg [24] reintroduced independently. Caserta et al. [4], Çakallı [3], Connor [9], Çolak [6], Et et al. [5, 11-13], Fridy [17], Salat [21], Altınok et al. [2], Çolak et al. [7, 8], and many others investigated some arguments related to this notion.

Çolak [6] studied statistical convergence order $\alpha$ by giving the definition as follows: we say that the sequence $x=\left(x_{k}\right)$ is statistically convergent of order $\alpha$ to L if there is a complex number L such that

$$
\lim _{n \rightarrow \infty} \frac{1}{n^{\alpha}}\left|\left\{k \leqslant n:\left|x_{k}-L\right| \geqslant \varepsilon\right\}\right|=0 .
$$

Let $0<\alpha \leqslant \beta \leqslant 1$. We define the $(\alpha, \beta)$-density of the subset $A$ of $\mathbb{N}$ (set of natural numbers) by

$$
\delta_{\alpha}^{\beta}(A)=\lim _{n} \frac{1}{n^{\alpha}}|\{k \leqslant n: k \in A\}|^{\beta}
$$

provided the limit exists (finite or infinite), where $|\{k \leqslant n: k \in A\}|^{\beta}$ denotes the $\beta$ th power of number of elements of $A$ not exceeding $n$.

Throughout this paper $w$ indicates the space of sequences of real number.

Email address: hacer.sengul@hotmail. com (Hacer Şengül) doi:10.22436/jnsa.010.09.46 
Let $0<\beta \leqslant 1,0<\alpha \leqslant 1, \alpha \leqslant \beta$, and $x=\left(x_{k}\right) \in w$. Space of sequences of $S_{\alpha}^{\beta}$-convergent (or statistically convergent sequences of order $(\alpha, \beta))$ is defined by

$$
S_{\alpha}^{\beta}=\left\{x=\left(x_{k}\right): \lim _{n \rightarrow \infty} \frac{1}{n^{\alpha}}\left|\left\{k \leqslant n:\left|x_{k}-L\right| \geqslant \varepsilon\right\}\right|^{\beta}=0\right\}
$$

where there exists a real number $L$. This convergence is indicated by $S_{\alpha}^{\beta}-\lim x_{k}=L$ (see [25]).

By a lacunary sequence we mean an increasing integer sequence $\theta=\left(k_{r}\right)$ such that $h_{r}=\left(k_{r}-k_{r-1}\right) \rightarrow$ $\infty$ as $r \rightarrow \infty$ and $\alpha \in(0,1]$. Through this paper the intervals determined by $\theta$ will be denoted by $I_{r}=\left(k_{r-1}, k_{r}\right]$ and the ratio $\frac{k_{r}}{k_{r-1}}$ will be abbreviated by $q_{r}$.

Subsequently lacunary sequences have been studied in ([1, 10, 16, 18-20, 22, 23]).

\section{Main results}

In this Section, we define the concepts of $S_{\alpha}^{\beta}(\theta)$-convergence and strong $N_{\alpha}^{\beta}(\theta, p)$-summability of sequences of complex (or real) numbers for $0<\alpha \leqslant \beta \leqslant 1$. Furthermore, we mention the inclusion relationships among the set of $S_{\alpha}^{\beta}(\theta)$-convergent sequences and the set of strongly $N_{\alpha}^{\beta}(\theta, p)$-summable sequences for different $\alpha$ and $\beta$ values.

Definition 2.1. Let $\theta=\left(k_{r}\right)$ be a lacunary sequence and $0<\alpha \leqslant \beta \leqslant 1$. $h_{r}^{\alpha}$ denotes the $\alpha$ th power $\left(h_{r}\right)^{\alpha}$ of $h_{r}$, and $|\{k \leqslant n: k \in A\}|^{\beta}$ denotes the $\beta$ th power of number of elements of $A$ not exceeding n. $S_{\alpha}^{\beta}(\theta)$ convergent (or lacunary statistically convergent sequences of order $(\alpha, \beta)$ ) sequences spaces is defined by

$$
S_{\alpha}^{\beta}(\theta)=\left\{x=\left(x_{k}\right): \lim _{r \rightarrow \infty} \frac{1}{h_{r}^{\alpha}}\left|\left\{k \in I_{r}:\left|x_{k}-L\right| \geqslant \varepsilon\right\}\right|^{\beta}=0\right\},
$$

where there exists a real number $L$. In the present case this convergence is indicated by $S_{\alpha}^{\beta}(\theta)-\lim x_{k}=L$. $S_{\alpha}^{\beta}(\theta)$ will indicate the set of all $S_{\alpha}^{\beta}(\theta)$-statistically convergent sequences. If $\theta=\left(2^{r}\right)$, then we will write $S_{\alpha}^{\beta}$ in the place of $S_{\alpha}^{\beta}(\theta)$. If $\alpha=\beta=1$ and $\theta=\left(2^{r}\right)$, then we will write $S$ in the place of $S_{\alpha}^{\beta}(\theta)$.

The $S_{\alpha}^{\beta}(\theta)$-convergent is in a good way defined for $\alpha \leqslant \beta$, but it is not usually in a good way defined for $\beta<\alpha$. Define $x=\left(x_{k}\right)$ by for $r=1,2, \ldots$,

$$
x_{k}=\left\{\begin{array}{lll}
1, & \text { if } k=2 r \\
0, & \text { if } k \neq 2 r
\end{array}\right.
$$

Then for every $\varepsilon>0$

$$
\lim _{r \rightarrow \infty} \frac{1}{h_{r}^{\alpha}}\left|\left\{k \in I_{r}:\left|x_{k}-1\right| \geqslant \varepsilon\right\}\right|^{\beta} \leqslant \lim _{r \rightarrow \infty} \frac{h_{r}^{\beta}}{2^{\beta} h_{r}^{\alpha}}=0
$$

and

$$
\lim _{r \rightarrow \infty} \frac{1}{h_{r}^{\alpha}}\left|\left\{k \in I_{r}:\left|x_{k}-0\right| \geqslant \varepsilon\right\}\right|^{\beta} \leqslant \lim _{r \rightarrow \infty} \frac{h_{r}^{\beta}}{2^{\beta} h_{r}^{\alpha}}=0
$$

for $\beta<\alpha$. So $S_{\alpha}^{\beta}(\theta)-\lim x_{k}=1$ and $S_{\alpha}^{\beta}(\theta)-\lim x_{k}=0$. But this is impossible.

Theorem 2.2. Let $0<\alpha \leqslant \beta \leqslant 1$ and $x=\left(x_{k}\right), y=\left(y_{k}\right)$ be sequences of real numbers, then

(i) if $S_{\alpha}^{\beta}(\theta)-\lim x_{k}=x_{0}$ and $c \in \mathbb{C}$, then $S_{\alpha}^{\beta}(\theta)-\lim \left(c x_{k}\right)=c x_{0}$;

(ii) if $S_{\alpha}^{\beta}(\theta)-\lim x_{k}=x_{0}$ and $S_{\alpha}^{\beta}(\theta)-\lim y_{k}=y_{0}$, then $S_{\alpha}^{\beta}(\theta)-\lim \left(x_{k}+y_{k}\right)=x_{0}+y_{0}$. 
Definition 2.3. Let $\theta=\left(k_{r}\right)$ be a lacunary sequence, $0<\alpha \leqslant \beta \leqslant 1$, and $p$ be a positive real number. Space of sequences of strongly $N_{\alpha}^{\beta}(\theta)$-summable (or strong $N(\theta, p)$-summability of order $(\alpha, \beta)$ ) is defined by

$$
N_{\alpha}^{\beta}(\theta)=\left\{x=\left(x_{k}\right): \lim _{r \rightarrow \infty} \frac{1}{h_{r}^{\alpha}}\left(\sum_{k \in I_{r}}\left|x_{k}-L\right|^{p}\right)^{\beta}=0\right\},
$$

where there exists a real number L. In the present case we write $N_{\alpha}^{\beta}(\theta, p)-\lim x_{k}=L$. $N_{\alpha}^{\beta}(\theta, p)$ will denote the set of all strongly $N(\theta, p)$-summable of order $(\alpha, \beta)$. If $\alpha=\beta=1$, then we will write $N(\theta, p)$ in the place of $N_{\alpha}^{\beta}(\theta, p)$. If $\theta=\left(2^{r}\right)$, then we will write $w_{\alpha}^{\beta}(p)$ in the place of $N_{\alpha}^{\beta}(\theta, p)$. If $L=0$, then we will write $w_{\alpha, 0}^{\beta}(p)$ in the place of $w_{\alpha}^{\beta}(p) . N_{\alpha, 0}^{\beta}(\theta, p)$ will denote the set of all strongly $N_{\theta}(p)$-summable of order $(\alpha, \beta)$ to 0 .

Theorem 2.4. Let $\theta=\left(k_{r}\right)$ be a lacunary sequence and $0<\alpha \leqslant \beta \leqslant 1$. If $\lim \inf _{\mathrm{r}} \mathrm{q}_{\mathrm{r}}>1$, then $w_{\alpha}^{\beta}(\mathrm{p})-\lim \mathrm{x}_{\mathrm{k}}=$ L implies $\mathrm{N}_{\alpha}^{\beta}(\theta, \mathrm{p})-\lim \mathrm{x}_{\mathrm{k}}=\mathrm{L}$.

Proof. If $\liminf \mathrm{q}_{\mathrm{r}} \mathrm{q}_{\mathrm{r}}>1$, then $1+\delta \leqslant \mathrm{q}_{\mathrm{r}}$ for all $\mathrm{r} \geqslant 1$, where there is a $\delta>0$. Then for $x \in w_{\alpha, 0}^{\beta}(p)$, we write

$$
\begin{aligned}
\tau_{r, \beta}^{\alpha}=\frac{1}{h_{r}^{\alpha}}\left(\sum_{i \in I_{r}}\left|x_{i}\right|^{p}\right)^{\beta} & =\frac{1}{h_{r}^{\alpha}}\left(\sum_{i=1}^{k_{r}}\left|x_{i}\right|^{p}-\sum_{i=1}^{k_{r-1}}\left|x_{i}\right|^{p}\right)^{\beta} \\
& \leqslant \frac{1}{h_{r}^{\alpha}}\left(\sum_{i=1}^{k_{r}}\left|x_{i}\right|^{p}\right)^{\beta}+\frac{1}{h_{r}^{\alpha}}\left(\sum_{i=1}^{k_{r-1}}\left|x_{i}\right|^{p}\right)^{\beta} \\
& =\frac{k_{r}^{\alpha}}{h_{r}^{\alpha}} \frac{1}{k_{r}^{\alpha}}\left(\sum_{i=1}^{k_{r}}\left|x_{i}\right|^{p}\right)^{\beta}+\frac{k_{r-1}^{\alpha}}{h_{r}^{\alpha}} \frac{1}{k_{r-1}^{\alpha}}\left(\sum_{i=1}^{k_{r-1}}\left|x_{i}\right|^{p}\right)^{\beta} .
\end{aligned}
$$

Since $h_{r}=k_{r}-k_{r-1}$, we have

$$
\frac{k_{r}^{\alpha}}{h_{r}^{\alpha}} \leqslant \frac{(1+\delta)^{\alpha}}{\delta^{\alpha}} \text { and } \frac{k_{r-1}^{\alpha}}{h_{r}^{\alpha}} \leqslant \frac{1}{\delta^{\alpha}} .
$$

The terms $\frac{1}{k_{r}^{\alpha}}\left(\sum_{i=1}^{k_{r}}\left|x_{i}\right|^{p}\right)^{\beta}$ and $\frac{1}{k_{r-1}^{\alpha}}\left(\sum_{i=1}^{k_{r-1}}\left|x_{i}\right|^{p}\right)^{\beta}$ both converge to 0 , and it follows that $\tau_{r, \beta}^{\alpha}$ converges to 0 . Hence $x \in N_{\alpha, 0}^{\beta}(\theta, p)$.

Theorem 2.5. Let $\theta=\left(k_{r}\right)$ be a lacunary sequence and $0<\alpha \leqslant \beta \leqslant 1$. If $\lim \sup _{r} \frac{k_{r}}{k_{r-1}^{\alpha}}<\infty$, then $N(\theta, p) \subset$ $w_{\alpha}^{\beta}(p)$.

Proof. The proof can be seen easily.

Theorem 2.6. If $x \in w_{\alpha}^{\beta} \cap N_{\alpha}^{\beta}(\theta)$ and $\lim \sup _{r} \frac{k_{r}}{k_{r-1}^{\alpha}}<\infty$, then $N_{\alpha}^{\beta}(\theta)-\lim x_{k}=w_{\alpha}^{\beta}-\lim x_{k}$.

Proof. Let $\mathrm{N}_{\alpha}^{\beta}(\theta)-\lim x_{k}=\mathrm{L}$ and $w_{\alpha}^{\beta}-\lim x_{k}=\mathrm{L}_{1}$, and assume that $\mathrm{L} \neq \mathrm{L}_{1}$. Since $\lim \sup _{\mathrm{r}} \frac{\mathrm{k}_{\mathrm{r}}}{\mathrm{k}_{\mathrm{r}-1}^{\alpha}}<\infty$ by Theorem 2.5, we have $N_{0}(\theta, p) \subset w_{\alpha, 0}^{\beta}(p)$. Since $\left(x-L_{1}\right) \in N_{0}(\theta, p)$, we get $\left(x-L_{1}\right) \in w_{\alpha, 0}^{\beta}(p)$ and thus $\frac{1}{t^{\alpha}}\left(\sum_{i=1}^{t}\left|x_{i}-L_{1}\right|\right)^{\beta} \rightarrow 0$. Then we have

$$
\frac{1}{\mathrm{t}^{\alpha}}\left(\sum_{i=1}^{\mathrm{t}}\left|\mathrm{x}_{\mathrm{i}}-\mathrm{L}_{1}\right|\right)^{\beta}+\frac{1}{\mathrm{t}^{\alpha}}\left(\sum_{i=1}^{\mathrm{t}}\left|\mathrm{x}_{\mathrm{i}}-\mathrm{L}\right|\right)^{\beta} \geqslant \frac{1}{\mathrm{t}^{\alpha}}\left(\sum_{i=1}^{\mathrm{t}}\left|\mathrm{x}_{\mathrm{i}}-\mathrm{L}_{1}\right|+\sum_{i=1}^{\mathrm{t}}\left|\mathrm{x}_{\mathrm{i}}-\mathrm{L}\right|\right)^{\beta} \geqslant \frac{1}{\mathrm{t}^{\alpha}}\left(\left|\mathrm{L}-\mathrm{L}_{1}\right|\right)^{\beta}>0 .
$$

This is a contradiction, and the proof is completed. 
Theorem 2.7. Let $0<\alpha_{1} \leqslant \alpha_{2} \leqslant \beta_{1} \leqslant \beta_{2} \leqslant 1$ and $\theta=\left(k_{r}\right)$ be a lacunary sequence. For $0<p<\infty$, $N_{\alpha_{1}}^{\beta_{2}}(\theta, p) \subset S_{\alpha_{2}}^{\beta_{1}}(\theta)$ and the inclusion is certain for some $\alpha_{1}, \alpha_{2}, \beta_{1}$, and $\beta_{2}$.

Proof. Let $x=\left(x_{k}\right) \in w$ and $\varepsilon>0$. We can write

$$
\begin{aligned}
\left(\sum_{k \in I_{r}}\left|x_{k}-\mathrm{L}\right|^{p}\right)^{\beta_{2}}=\left(\sum_{\substack{k \in I_{r} \\
\left|x_{k}-\mathrm{L}\right| \geqslant \varepsilon}}\left|x_{k}-\mathrm{L}\right|^{p}+\sum_{\substack{k \in I_{r} \\
\left|x_{k}-\mathrm{L}\right|<\varepsilon}}\left|x_{k}-\mathrm{L}\right|^{p}\right)^{\beta_{2}} & \geqslant\left(\sum_{\substack{k \in I_{r} \\
\left|x_{k}-\mathrm{L}\right| \geqslant \varepsilon}}\left|x_{k}-\mathrm{L}\right|^{p}\right)^{\beta_{2}} \\
& \geqslant\left|\left\{k \in \mathrm{I}_{\mathrm{r}}:\left|x_{k}-\mathrm{L}\right| \geqslant \varepsilon\right\}\right|^{\beta_{2}} \varepsilon^{p \beta_{2}}
\end{aligned}
$$

and so that

$$
\frac{1}{h_{r}^{\alpha_{1}}}\left(\sum_{k \in I_{r}}\left|x_{k}-L\right|^{p}\right)^{\beta_{2}} \geqslant \frac{1}{h_{r}^{\alpha_{1}}}\left|\left\{k \in I_{r}:\left|x_{k}-L\right| \geqslant \varepsilon\right\}\right|^{\beta_{2}} \varepsilon^{p \beta_{2}} \geqslant \frac{1}{h_{r}^{\alpha_{2}}}\left|\left\{k \in I_{r}:\left|x_{k}-L\right| \geqslant \varepsilon\right\}\right|^{\beta_{1}} \varepsilon^{p \beta_{1}} .
$$

Hence $N_{\alpha_{1}}^{\beta_{2}}(\theta, p) \subset S_{\alpha_{2}}^{\beta_{1}}(\theta)$ for $0<\alpha_{1} \leqslant \alpha_{2} \leqslant \beta_{1} \leqslant \beta_{2} \leqslant 1$.

We demonstrate the certainty of the inclusion $N_{\alpha_{1}}^{\beta_{2}}(\theta, p) \subset S_{\alpha_{2}}^{\beta_{1}}(\theta)$ for $p=1$ and $0<\alpha_{1} \leqslant \alpha_{2} \leqslant \beta_{1} \leqslant$ $\beta_{2} \leqslant 1$. Define $x=\left(x_{k}\right)$ by

$$
x_{k}= \begin{cases}{\left[\sqrt{h_{r}}\right],} & k_{r-1}<k \leqslant k_{r}-1+\left[\sqrt{h_{r}}\right] \\ 0, & \text { otherwise. }\end{cases}
$$

We get for $\frac{1}{4}<\alpha_{2}<1$ and $\beta_{1}=\frac{1}{2}$,

$$
\frac{1}{h_{r}^{\alpha_{2}}}\left|\left\{k \in I_{r}:\left|x_{k}-0\right| \geqslant \varepsilon\right\}\right|^{\beta_{1}}=\frac{\left(\left[\sqrt{h_{r}}\right]\right)^{\beta_{1}}}{h_{r}^{\alpha_{2}}} \rightarrow 0, \text { as } r \rightarrow \infty,
$$

i.e., $S_{\alpha_{2}}^{\beta_{1}}(\theta)-\lim x_{k}=0$. For $0<\alpha_{1}<\frac{1}{4}$ and $\beta_{2}=1$,

$$
\frac{1}{h_{r}^{\alpha_{1}}}\left(\sum_{k \in I_{r}}\left|x_{k}\right|\right)^{\beta_{2}}=\frac{\left(\left[\sqrt{h_{r}}\right]\left[\sqrt{h_{r}}\right]\right)^{\beta_{2}}}{h_{r}^{\alpha_{1}}} \rightarrow \infty .
$$

For $0<\alpha_{1}<\frac{1}{4}$ and $\beta_{2}=1, N_{\alpha_{1}}^{\beta_{2}}(\theta, p)-\lim x_{k} \neq 0$. Therefore, $N_{\alpha_{1}}^{\beta_{2}}(\theta, p) \subset S_{\alpha_{2}}^{\beta_{1}}(\theta)$ is certain for $0<\alpha_{1}<$ $\frac{1}{4}, \beta_{2}=1, \frac{1}{4}<\alpha_{2}<1$, and $\beta_{1}=\frac{1}{2}$.

The following result is a consequence of Theorem 2.7.

Corollary 2.8. Let $0<\alpha_{1} \leqslant \alpha_{2} \leqslant \beta_{1} \leqslant \beta_{2} \leqslant 1$ and $0<p<\infty$.

i) If $\beta_{2}=1$, then $\mathrm{N}_{\alpha_{1}}(\theta, p) \subset S_{\alpha_{2}}^{\beta_{1}}(\theta)$ for $0<\alpha_{1} \leqslant \alpha_{2} \leqslant \beta_{1} \leqslant 1$.

ii) If $\beta_{1}=\beta_{2}=1$, then $N_{\alpha_{1}}(\theta, p) \subset S_{\alpha_{2}}(\theta)$ for $0<\alpha_{1} \leqslant \alpha_{2} \leqslant 1$.

Theorem 2.9. Let $0<\alpha \leqslant \beta \leqslant 1$ and $\theta=\left(k_{r}\right)$ be a lacunary sequence. If $\liminf _{\mathrm{r}} \mathrm{q}_{\mathrm{r}}>1$, then $\mathrm{S}_{\alpha}^{\beta} \subset \mathrm{S}_{\alpha}^{\beta}(\theta)$.

Proof. If lim inf $\mathrm{q}_{\mathrm{r}}>1$, then $\mathrm{q}_{\mathrm{r}} \geqslant 1+\delta$ for sufficiently large $\mathrm{r}$ where there is a $\delta>0$. We can write

$$
\frac{h_{r}}{k_{r}} \geqslant \frac{\delta}{1+\delta} \Longrightarrow \frac{1}{k_{r}^{\alpha}} \geqslant \frac{\delta^{\alpha}}{(1+\delta)^{\alpha}} \frac{1}{h_{r}^{\alpha}} \text {. }
$$

If $S_{\alpha}^{\beta}-\lim x_{k}=L$, then for every $\varepsilon>0$, we have

$$
\frac{1}{\mathrm{k}_{\mathrm{r}}^{\alpha}}\left|\left\{\mathrm{k} \leqslant \mathrm{k}_{\mathrm{r}}:\left|x_{\mathrm{k}}-\mathrm{L}\right| \geqslant \varepsilon\right\}\right|^{\beta} \geqslant \frac{1}{\mathrm{k}_{\mathrm{r}}^{\alpha}}\left|\left\{\mathrm{k} \in \mathrm{I}_{\mathrm{r}}:\left|\mathrm{x}_{\mathrm{k}}-\mathrm{L}\right| \geqslant \varepsilon\right\}\right|^{\beta} \geqslant \frac{\delta^{\alpha}}{(1+\delta)^{\alpha}} \frac{1}{\mathrm{~h}_{\mathrm{r}}^{\alpha}}\left|\left\{\mathrm{k} \in \mathrm{I}_{\mathrm{r}}:\left|x_{\mathrm{k}}-\mathrm{L}\right| \geqslant \varepsilon\right\}\right|^{\beta} .
$$

Therefore $S_{\alpha}^{\beta}-\lim x_{k}=L$ implies $S_{\alpha}^{\beta}(\theta)-\lim x_{k}=L$. 
Theorem 2.10. Let $0<\alpha_{1} \leqslant \alpha_{2} \leqslant \beta_{1} \leqslant \beta_{2} \leqslant 1$. If

$$
\lim _{r \rightarrow \infty} \inf \left(\frac{h_{r}}{k_{r}}\right)^{\alpha_{2}}>0
$$

then $S_{\alpha_{1}} \subset S_{\alpha_{2}}^{\beta_{1}}(\theta)$.

Proof. For a given $\varepsilon>0$, we have

$$
\left\{k \leqslant k_{r}:\left|x_{k}-L\right| \geqslant \varepsilon\right\} \supset\left\{k \in I_{r}:\left|x_{k}-L\right| \geqslant \varepsilon\right\} .
$$

Therefore,

$$
\frac{1}{k_{r}^{\alpha_{1}}}\left|\left\{k \leqslant k_{r}:\left|x_{k}-L\right| \geqslant \varepsilon\right\}\right| \geqslant \frac{1}{k_{r}^{\alpha_{2}}}\left|\left\{k \in I_{r}:\left|x_{k}-L\right| \geqslant \varepsilon\right\}\right|^{\beta_{2}} \geqslant \frac{h_{r}^{\alpha_{2}}}{k_{r}^{\alpha_{2}}} \frac{1}{h_{r}^{\alpha_{2}}}\left|\left\{k \in I_{r}:\left|x_{k}-L\right| \geqslant \varepsilon\right\}\right|^{\beta_{1}} .
$$

Since $\lim _{r \rightarrow \infty} \inf \left(\frac{h_{r}}{k_{r}}\right)^{\alpha_{2}}>0$ and $x=\left(x_{k}\right) \in S_{\alpha_{1}}$, we have $x \in S_{\alpha_{2}}^{\beta_{1}}(\theta)$.

Theorem 2.11. Let $\theta=\left(k_{\mathrm{r}}\right)$ and $\theta^{\prime}=\left(\mathrm{s}_{\mathrm{r}}\right)$ be two lacunary sequences such that $\mathrm{I}_{\mathrm{r}} \subset \mathrm{J}_{\mathrm{r}}$ for all $\mathrm{r} \in \mathbb{N}$ and $0<\alpha_{1} \leqslant \alpha_{2} \leqslant \beta_{1} \leqslant \beta_{2} \leqslant 1$,

(i) if

$$
\lim _{\mathrm{r} \rightarrow \infty} \inf \frac{h_{\mathrm{r}}^{\alpha_{1}}}{\ell_{\mathrm{r}}^{\alpha_{2}}}>0
$$

then $S_{\alpha_{2}}^{\beta_{2}}\left(\theta^{\prime}\right) \subseteq S_{\alpha_{1}}^{\beta_{1}}(\theta)$;

(ii) if

$$
\lim _{r \rightarrow \infty} \frac{\ell_{r}}{h_{r}^{\alpha_{2}}}=1
$$

then $S_{\alpha_{1}}^{\beta_{2}}(\theta) \subseteq S_{\alpha_{2}}^{\beta_{1}}\left(\theta^{\prime}\right)$.

Proof.

(i). Suppose that $I_{r} \subset J_{r}$ for all $r \in \mathbb{N}$. We can write

$$
\left\{k \in J_{r}:\left|x_{k}-L\right| \geqslant \varepsilon\right\} \supseteq\left\{k \in I_{r}:\left|x_{k}-L\right| \geqslant \varepsilon\right\}
$$

and so

$$
\frac{1}{\ell_{\mathrm{r}}^{\alpha_{2}}}\left|\left\{k \in \mathrm{J}_{\mathrm{r}}:\left|x_{\mathrm{k}}-\mathrm{L}\right| \geqslant \varepsilon\right\}\right|^{\beta_{2}} \geqslant \frac{\mathrm{h}_{\mathrm{r}}^{\alpha_{1}}}{\ell_{\mathrm{r}}^{\alpha_{2}}} \frac{1}{h_{\mathrm{r}}^{\alpha_{1}}}\left|\left\{k \in \mathrm{I}_{\mathrm{r}}:\left|x_{\mathrm{k}}-\mathrm{L}\right| \geqslant \varepsilon\right\}\right|^{\beta_{1}}
$$

for all $r \in \mathbb{N}$, where $I_{r}=\left(k_{r-1}, k_{r}\right], J_{r}=\left(s_{r-1}, s_{r}\right], h_{r}=k_{r}-k_{r-1}, \ell_{r}=s_{r}-s_{r-1}$. Thus we get $S_{\alpha_{2}}^{\beta_{2}}\left(\theta^{\prime}\right) \subseteq S_{\alpha_{1}}^{\beta_{1}}(\theta)$.

(ii). Let $x=\left(x_{k}\right) \in S_{\alpha_{1}}^{\beta_{2}}(\theta)$ and (2.2) be satisfied. Since $I_{r} \subset J_{r}$, for $\varepsilon>0$ we may write

$$
\begin{aligned}
\frac{1}{\ell_{r}^{\alpha_{2}}}\left|\left\{k \in J_{r}:\left|x_{k}-L\right| \geqslant \varepsilon\right\}\right|^{\beta_{1}}= & \frac{1}{\ell_{r}^{\alpha_{2}}}\left|\left\{s_{r-1}<k \leqslant k_{r-1}:\left|x_{k}-L\right| \geqslant \varepsilon\right\}\right|^{\beta_{1}} \\
& +\frac{1}{\ell_{r}^{\alpha_{2}}}\left|\left\{k_{r}<k \leqslant s_{r}:\left|x_{k}-L\right| \geqslant \varepsilon\right\}\right|^{\beta_{1}}+\frac{1}{\ell_{r}^{\alpha_{2}}}\left|\left\{k_{r-1}<k \leqslant k_{r}:\left|x_{k}-L\right| \geqslant \varepsilon\right\}\right|^{\beta_{1}} \\
\leqslant & \frac{1}{\ell_{r}^{\alpha_{2}}}\left(k_{r-1}-s_{r-1}\right)^{\beta_{1}}+\frac{1}{\ell_{r}^{\alpha_{2}}}\left(s_{r}-k_{r}\right)^{\beta_{1}}+\frac{1}{\ell_{r}^{\alpha_{2}}}\left|\left\{k \in I_{r}:\left|x_{k}-L\right| \geqslant \varepsilon\right\}\right|^{\beta_{1}} \\
\leqslant & \frac{k_{r-1}-s_{r-1}}{\ell_{r}^{\alpha_{2}}}+\frac{s_{r}-k_{r}}{\ell_{r}^{\alpha_{2}}}+\frac{1}{\ell_{r}^{\alpha_{2}}}\left|\left\{k \in I_{r}:\left|x_{k}-L\right| \geqslant \varepsilon\right\}\right|^{\beta_{1}}
\end{aligned}
$$




$$
\begin{aligned}
& =\frac{\ell_{\mathrm{r}}-h_{\mathrm{r}}}{\ell_{\mathrm{r}}^{\alpha_{2}}}+\frac{1}{\ell_{\mathrm{r}}^{\alpha_{2}}}\left|\left\{k \in \mathrm{I}_{\mathrm{r}}:\left|x_{\mathrm{k}}-\mathrm{L}\right| \geqslant \varepsilon\right\}\right|^{\beta_{1}} \\
& \leqslant \frac{\ell_{\mathrm{r}}-h_{r}^{\alpha_{2}}}{h_{r}^{\alpha_{2}}}+\frac{1}{h_{r}^{\alpha_{1}}}\left|\left\{k \in \mathrm{I}_{\mathrm{r}}:\left|x_{\mathrm{k}}-\mathrm{L}\right| \geqslant \varepsilon\right\}\right|^{\beta_{2}} \\
& \leqslant\left(\frac{\ell_{\mathrm{r}}}{h_{\mathrm{r}}^{\alpha_{2}}}-1\right)+\frac{1}{h_{r}^{\alpha_{1}}}\left|\left\{k \in \mathrm{I}_{\mathrm{r}}:\left|x_{k}-\mathrm{L}\right| \geqslant \varepsilon\right\}\right|^{\beta_{2}}
\end{aligned}
$$

for all $r \in \mathbb{N}$. This implies that $S_{\alpha_{1}}^{\beta_{2}}(\theta) \subseteq S_{\alpha_{2}}^{\beta_{1}}\left(\theta^{\prime}\right)$.

Theorem 2.12. Let $\theta=\left(k_{r}\right)$ and $\theta^{\prime}=\left(s_{r}\right)$ be two lacunary sequences such that $\mathrm{I}_{\mathrm{r}} \subseteq \mathrm{J}_{\mathrm{r}}$ for all $\mathrm{r} \in \mathbb{N}, 0<\alpha_{1} \leqslant$ $\alpha_{2} \leqslant \beta_{1} \leqslant \beta_{2} \leqslant 1$, and $0<p<\infty$. Then we have

(i) if (2.1) is provided, then $w_{\alpha_{2}}^{\beta_{2}}\left(\theta^{\prime}, p\right) \subset w_{\alpha_{1}}^{\beta_{1}}(\theta, p)$;

(ii) if (2.2) is provided and $x \in \ell_{\infty}$, then $w_{\alpha_{1}}^{\beta_{2}}(\theta, p) \subset w_{\alpha_{2}}^{\beta_{1}}\left(\theta^{\prime}, p\right)$;

(iii) if (2.1) is provided, then $w_{\alpha_{2}}^{\beta_{2}}\left(\theta^{\prime}, p\right) \subset S_{\alpha_{1}}^{\beta_{1}}(\theta)$;

(iv) if (2.2) is provided and $x \in \ell_{\infty}$, then $S_{\alpha_{1}}^{\beta_{2}}(\theta) \subset w_{\alpha_{2}}^{\beta_{1}}\left(\theta^{\prime}, p\right)$.

Proof.

(i). The proof can be seen easily.

(ii). Let $x=\left(x_{k}\right) \in w_{\alpha_{1}}^{\beta_{2}}(\theta, p)$ and assume that (2.2) is provided. Since $x=\left(x_{k}\right) \in \ell_{\infty}$, then we obtain $\left|x_{k}-L\right| \leqslant M$ for all $k$ where there is an $M>0$. Now, since $I_{r} \subseteq J_{r}$ and $h_{r} \leqslant \ell_{r}$ for all $r \in \mathbb{N}$, we may write

$$
\begin{aligned}
\frac{1}{\ell_{r}^{\alpha_{2}}}\left(\sum_{k \in J_{r}}\left|x_{k}-L\right|^{p}\right)^{\beta_{1}} & =\frac{1}{\ell_{r}^{\alpha_{2}}}\left(\sum_{k \in J_{r}-I_{r}}\left|x_{k}-L\right|^{p}\right)^{\beta_{1}}+\frac{1}{\ell_{r}^{\alpha_{2}}}\left(\sum_{k \in I_{r}}\left|x_{k}-L\right|^{p}\right)^{\beta_{1}} \\
& \leqslant\left(\frac{\ell_{r}-h_{r}}{\ell_{r}^{\alpha_{2}}}\right)^{\beta_{1}} M^{p \beta_{1}}+\frac{1}{\ell_{r}^{\alpha_{2}}}\left(\sum_{k \in I_{r}}\left|x_{k}-L\right|^{p}\right)^{\beta_{1}} \\
& \leqslant\left(\frac{\ell_{r}-h_{r}^{\alpha_{2}}}{h_{r}^{\alpha_{2}}}\right) M^{p \beta_{1}}+\frac{1}{h_{r}^{\alpha_{2}}}\left(\sum_{k \in I_{r}}\left|x_{k}-L\right|^{p}\right)^{\beta_{2}} \\
& \leqslant\left(\frac{\ell_{r}}{h_{r}^{\alpha_{2}}}-1\right) M^{p \beta_{1}}+\frac{1}{h_{r}^{\alpha_{1}}}\left(\sum_{k \in I_{r}}\left|x_{k}-L\right|^{p}\right)^{\beta_{2}}
\end{aligned}
$$

for every $r \in \mathbb{N}$. Therefore $\ell_{\infty} \cap w_{\alpha_{1}}^{\beta_{2}}(\theta, p) \subset w_{\alpha_{2}}^{\beta_{1}}\left(\theta^{\prime}, p\right)$.

(iii). Let $x=\left(x_{k}\right) \in w_{\alpha_{2}}^{\beta_{2}}\left(\theta^{\prime}, p\right)$ and $\varepsilon>0$, we can write

$$
\begin{aligned}
& \left(\sum_{k \in J_{r}}\left|x_{k}-L\right|^{p}\right)^{\beta_{2}}=\left(\sum_{\substack{k \in J r \\
\left|x_{k}-L\right| \geqslant \varepsilon}}\left|x_{k}-L\right|^{p}+\sum_{\substack{k \in r_{r} \\
\left|x_{k}-L\right|<\varepsilon}}\left|x_{k}-L\right|^{p}\right)^{\beta_{2}} \\
& \geqslant\left(\sum_{\substack{k \in I_{r} \\
\left|x_{k}-L\right| \geqslant \varepsilon}}\left|x_{k}-L\right|^{p}+\sum_{\substack{k \in I_{r} \\
\left|x_{k}-L\right|<\varepsilon}}\left|x_{k}-L\right|^{p}\right)^{\beta_{1}}
\end{aligned}
$$




$$
\geqslant\left(\sum_{\substack{k \in I_{r} \\\left|x_{k}-L\right| \geqslant \varepsilon}}\left|x_{k}-L\right|^{p}\right)^{\beta_{1}} \geqslant\left|\left\{k \in I_{r}:\left|x_{k}-L\right| \geqslant \varepsilon\right\}\right|^{\beta_{1}} \varepsilon^{p \beta_{1}}
$$

and so that

$$
\frac{1}{\ell_{r}^{\alpha_{2}}}\left(\sum_{k \in J_{r}}\left|x_{k}-L\right|^{p}\right)^{\beta_{2}} \geqslant \frac{1}{\ell_{r}^{\alpha_{2}}}\left|\left\{k \in I_{r}:\left|x_{k}-L\right| \geqslant \varepsilon\right\}\right|^{\beta_{1}} \varepsilon^{p \beta_{1}} \geqslant \frac{h_{r}^{\alpha_{1}}}{\ell_{r}^{\alpha_{2}}} \frac{1}{h_{r}^{\alpha_{1}}}\left|\left\{k \in I_{r}:\left|x_{k}-L\right| \geqslant \varepsilon\right\}\right|^{\beta_{1}} \varepsilon^{p \beta_{1}} .
$$

Therefore $x \in S_{\alpha_{1}}^{\beta_{1}}(\theta)$.

(iv). Assume that $x=\left(x_{k}\right) \in S_{\alpha_{1}}^{\beta_{2}}(\theta)$ and $x \in \ell_{\infty}$. Then we obtain $\left|x_{k}-L\right| \leqslant M$ for all $k$ where there is an $M>0$. We may write

$$
\begin{aligned}
& \frac{1}{\ell_{r}^{\alpha_{2}}}\left(\sum_{k \in J_{r}}\left|x_{k}-L\right|^{p}\right)^{\beta_{1}}=\frac{1}{\ell_{r}^{\alpha_{2}}}\left(\sum_{k \in J_{r}-I_{r}}\left|x_{k}-L\right|^{p}\right)^{\beta_{1}}+\frac{1}{\ell_{r}^{\alpha_{2}}}\left(\sum_{k \in I_{r}}\left|x_{k}-L\right|^{p}\right)^{\beta_{1}} \\
& \leqslant\left(\frac{\ell_{\mathrm{r}}-h_{\mathrm{r}}}{\ell_{\mathrm{r}}^{\alpha_{2}}}\right) M^{\mathrm{p} \beta_{1}}+\frac{1}{\ell_{\mathrm{r}}^{\alpha_{2}}}\left(\sum_{k \in I_{r}}\left|x_{k}-\mathrm{L}\right|^{p}\right)^{\beta_{1}} \\
& \leqslant\left(\frac{\ell_{\mathrm{r}}-h_{\mathrm{r}}^{\alpha_{2}}}{\ell_{\mathrm{r}}^{\alpha_{2}}}\right) M^{p \beta_{1}}+\frac{1}{\ell_{r}^{\alpha_{2}}}\left(\sum_{k \in I_{r}}\left|x_{k}-L\right|^{p}\right)^{\beta_{2}} \\
& \leqslant\left(\frac{\ell_{\mathrm{r}}}{h_{\mathrm{r}}^{\alpha_{2}}}-1\right) M^{p \beta_{1}}+\frac{1}{h_{r}^{\alpha_{1}}}\left(\sum_{\substack{k \in \mathrm{I}_{\mathrm{r}} \\
\left|x_{\mathrm{k}}-\mathrm{L}\right| \geqslant \varepsilon}}\left|x_{\mathrm{k}}-\mathrm{L}\right|^{p}\right)^{\beta_{2}}+\frac{1}{h_{\mathrm{r}}^{\alpha_{2}}}\left(\sum_{\substack{k \in I_{\mathrm{r}} \\
\left|x_{\mathrm{k}}-\mathrm{L}\right|<\varepsilon}}\left|x_{k}-\mathrm{L}\right|^{p}\right)^{\beta_{2}} \\
& \leqslant\left(\frac{\ell_{\mathrm{r}}}{h_{\mathrm{r}}^{\alpha_{2}}}-1\right) M^{p \beta_{1}}+\frac{M^{p \beta_{2}}}{h_{r}^{\alpha_{1}}}\left|\left\{k \in I_{r}:\left|x_{k}-L\right| \geqslant \varepsilon\right\}\right|^{\beta_{2}}+\frac{h_{r}^{\beta_{2}}}{h_{r}^{\alpha_{2}}} \varepsilon^{p \beta_{2}} \\
& \leqslant\left(\frac{\ell_{r}}{h_{r}^{\alpha_{2}}}-1\right) M^{p \beta_{1}}+\frac{M^{p \beta_{2}}}{h_{r}^{\alpha_{1}}}\left|\left\{k \in I_{r}:\left|x_{k}-L\right| \geqslant \varepsilon\right\}\right|^{\beta_{2}}+\frac{\ell_{r}}{h_{r}^{\alpha_{2}}} \varepsilon^{p \beta_{2}}
\end{aligned}
$$

for every $\varepsilon>0$ and all $r \in \mathbb{N}$. Thus $x=\left(x_{k}\right) \in w_{\alpha_{2}}^{\beta_{1}}\left(\theta^{\prime}, p\right)$.

\section{Conclusion}

In this paper, the set of $S^{\alpha}(\theta)$-convergent sequences and the set of strongly $N^{\alpha}(\theta, p)$-summable sequences were generalized to the sets of order $(\alpha, \beta)$. We can obtain different inclusions from Theorems 2.10, 2.11, and 2.12 for $\beta_{2}=1, \beta_{1}=\beta_{2}=1, \alpha_{2}=\beta_{1}=\beta_{2}=1, \beta_{1}=\beta_{2}$, and $\alpha_{1}=\alpha_{2}$. If we look closely, then we have the set of $S^{\alpha}(\theta)$-convergent sequences and the set of strongly $N^{\alpha}(\theta, p)$-summable sequences for $\beta_{1}=\beta_{2}=1$.

\section{Acknowledgment}

I am very much thankful to the referees for their valuable comments on this article and to the dear editor due to his help. 


\section{References}

[1] H. Altınok, Y. Altın, M. Et, Lacunary almost statistical convergence of fuzzy numbers, Thai J. Math., 2 (2004), $265-274$. 1

[2] H. Altınok, Y. Altın, M. Işık, The sequence space $\mathrm{BV}_{\sigma}(M, p, q, s)$ on seminormed spaces, Indian J. Pure Appl. Math., 39 (2008), 49-58. 1

[3] H. Çakalli, A study on statistical convergence, Funct. Anal. Approx. Comput., 1 (2009), 19-24. 1

[4] A. Caserta, G. Di Maio, L. D. R. Kočinac, Statistical convergence in function spaces, Abstr. Appl. Anal., 2011 (2011), 11 pages. 1

[5] M. Çınar, M. Karakaş, M. Et, On pointwise and uniform statistical convergence of order $\alpha$ for sequences of functions, Fixed Point Theory Appl., 2013 (2013), 11 pages. 1

[6] R. Çolak, Statistical convergence of order $\alpha$, Modern Methods in Analysis and Its Applications, 2010 (2010), 9 pages. 1

[7] R. Çolak, Y. Altın, M. Et, $\lambda$-almost statistical convergence of order $\alpha$, An. Ştiinţ. Univ. Al. I. Cuza Iaşi. Mat. (N.S.), 60 (2014), 437-448. 1

[8] R. Çolak, Ç. A. Bektaş, 入-statistical convergence of order $\alpha$, Acta Math. Sci. Ser. B Engl. Ed., 31 (2011), 953-959. 1

[9] J. S. Connor, The Statistical and Strong p-Cesaro Convergence of Sequences, Analysis, 8 (1988), 47-63. 1

[10] G. Das, S. K. Mishra, Banach limits and lacunary strong almost convergence, J. Orissa Math. Soc., 2 (1983), 61-70. 1

[11] M. Et, Generalized Cesàro difference sequence spaces of non-absolute type involving lacunary sequences, Appl. Math. Comput., 219 (2013), 9372-9376. 1

[12] M. Et, H. Altınok, Y. Altin, On some generalized sequence spaces, Appl. Math. Comput., 154 (2004), 167-173.

[13] M. Et, M. Çınar, M. Karakaş, On $\lambda$-statistical convergence of order $\alpha$ of sequences of function, J. Inequal. Appl., 2013 (2013), 8 pages. 1

[14] M. Et, H. Şengül, Some Cesaro-type summability spaces of order $\alpha$ and lacunary statistical convergence of order $\alpha$, Filomat, 28 (2014), 1593-1602.

[15] H. Fast, Sur La Convergence Statistique, Colloquium Math., 2 (1951), 241-244. 1

[16] A. R. Freedman, J. J. Sember, M. Raphael, Some Cesaro-type summability spaces, Proc. London Math. Soc., 37 (1978), 508-520. 1

[17] J. Fridy, On Statistical Convergence, Analysis, 5 (1985), 301-313. 1

[18] J. A. Fridy, C. Orhan, Lacunary Statistical Convergence, Pacific J. Math., 160 (1993), 43-51. 1

[19] J. A. Fridy, C. Orhan, Lacunary Statistical Summability, J. Math. Anal. Appl, 173 (1993), 497-504.

[20] C. Mongkolkeha, P. Kumam, Some geometric properties of lacunary sequence spaces related to fixed point property, Abstr. Appl. Anal., 2011 (2011), 13 pages. 1

[21] T. Salát, On Statistically Convergent Sequences of Real Numbers, Math. Slovaca, 30 (1980), 139-150. 1

[22] E. Savaş, On I-lacunary statistical convergence of order $\alpha$ for sequences of sets, Filomat, 29 (2015), 1223-1229. 1

[23] R. Savaş Eren, E. Savaş, Double lacunary statistical convergence of order $\alpha$, Indian J. Math., 57 (2015), 1-15. 1

[24] I. J. Schoenberg, The Integrability of Certain Functions and Related Summability Methods, Amer. Math. Monthly, 66 (1959), 361-375. 1

[25] H. Şengül, Some Cesro-type summability spaces defined by a modulus function of order $(\alpha, \beta)$, Commun. Fac. Sci. Univ. Ank. Sr. A1 Math. Stat., 66 (2017), 80-90. 1

[26] H. Şengül, M. Et, On lacunary statistical convergence of order $\alpha$, Acta Math. Sci. Ser. B Engl. Ed., 34 (2014), $473-482$.

[27] H. Steinhaus, Sur la convergence ordinaire et la convergence asymptotique, Colloq. Math., 2 (1951), 73-74. 1 